\title{
Análisis de calidad de servicio en la transmisión por AM digital mediante el uso de códecs en USRP.
}

\author{
Analysis of quality of service in digital AM transmission through the use of \\ codecs in USRP
}

Juan José Pérez Insuasti. ${ }^{1}$, Mónica Andrea Zabala Haro. ${ }^{2}$, Alexandra Orfelina Pazmiño Armijos. ${ }^{3}$ Hugo Oswaldo Moreno Avilés. ${ }^{4}$

Recibido: 10-02-2020 / Revisado: 15-03-2020 /Aceptado: 14-04-2020/ Publicado: 4-06-2020

\begin{abstract}
.
DOI: https://doi.org/10.33262/concienciadigital.v3i2.2.1254

The objective was to conduct the study and analysis of the quality of service in digital transmission through the use of codecs in USRP. The development methodology defines the realization of the prototype for transmission and reception, starting with the selection of devices, the design, execution, and analysis of the results obtained. It was made the study of the particular conditions in the transmission and reception of the signal in digital AM (DRM), the implementation of the transmitter in GNU Radio, the configuration of the receiver in HDSDR and Dream SDR. In addition to the cards Ettus USRP N210, Ettus USRP B200, and RTL-SDR REALTEK RTL2832U. The result of this study was the quality of the service evaluated with respect to the transmission speed (Kbps) and the signal-to-noise ratio (SNR) to determine the fidelity in the reception of digital signals. Based on the results defined in the operating characteristics of each mode of robustness for a given transmission channel vary the values of the transmission speed and the signal-to-noise ratio, offering B mode better signal protection with respect to noise, while A mode offers higher transmission speed. Therefore, it is concluded that the DRM system uses the best sound compression technologies since it

\footnotetext{
${ }^{1}$ Instituto Superior Tecnológico Carlos Cisneros, Riobamba, Ecuador, juanjos19_19@yahoo.es

${ }^{2}$ Escuela Superior Politécnica de Chimborazo, Facultad de Informática y Electrónica. Riobamba, Ecuador.m_zabala@espoch.edu.ec

${ }^{3}$ Escuela Superior Politécnica de Chimborazo. Riobamba, Ecuador. apazmino_a@espoch.edu.ec

${ }^{4}$ Escuela Superior Politécnica de Chimborazo, Facultad de Informática y Electrónica. Riobamba, Ecuador. h_moreno@espoch.edu.ec
} 
uses the MPEG-4 audio and voice algorithms that provide excellent sound quality at low bit rates with advanced audio coding (AAC).

Keywords: Radio Communications, Digital Signal Transmission, Modulated Amplitude (Am), World Digital Radio (Drm), Transmission Speed, Noise Signal Relation (Snr), Defined Radio By Software (Sdr).

\section{Resumen.}

El objetivo fue realizar el estudio y análisis de la calidad de servicio en la transmisión am digital mediante el uso de códecs en USRP. La Metodología de desarrollo muestra claramente cómo se realizó el prototipo para la transmisión y recepción, empezando con la selección de dispositivos, el diseño, la ejecución y el análisis de los resultados obtenidos. Se basa en el estudio de las condiciones particulares en la transmisión y recepción de señal en AM digital (DRM), la implementación del transmisor en GNU Radio, la configuración del receptor en HDSDR y Dream SDR. Además de las tarjetas Ettus USRP N210, Ettus USRP B200 y RTL-SDR REALTEK RTL2832U. El resultado de este estudio fue la calidad de los niveles de servicio evaluados en la velocidad de transmisión (Kbps) y la relación señal / ruido (SNR) para determinar la fidelidad en la recepción de señales digitales. En base a los resultados se define que las características de funcionamiento de cada modo de robustez para un determinado canal de transmisión varían los valores de la velocidad de transmisión y la relación señal a ruido, ofreciendo el modo $\mathrm{B}$ una mejor protección de la señal con respecto al ruido, mientras que el modo A ofrece mayor velocidad de transmisión. El sistema DRM emplea las mejores tecnologías de compresión de sonido ya que usa los algoritmos de audio y voz del MPEG-4 que brinda excelente calidad de sonido a bajas tasas de bit como la codificación AAC.

Palabras claves: Transmisión de Señales Digitales, Amplitud Modulada (Am), Digital Radio Mondiale (Drm), Velocidad de Transmision, Relacion Señal a Ruido (Srn), Radio Definida Por Software (Sdr).

\section{Introducción.}

DRM es un estándar para la transmisión de señales digitales de radiodifusión, definido inicialmente para las frecuencias menores a $30 \mathrm{MHz}$ en las bandas de LF, MF y HF, se lo denomina también como DRM30. (Gil \& Santillán, 2015; Puente, 2005)

El sistema permite tener una mejor calidad de sonido similar a las estaciones de radiodifusión en FM comerciales, mejora la distribución del ancho de banda y efectos multitrayectoria 
gracias a que utiliza la codificación OFDM. Para cubrir la banda VHF de transmisiones de señal en FM se incorpora el estándar DRM+, una variante de DRM para estas frecuencias. (Erazo, 2009; Salinas, 2011; Sánchez \& Sagbay, 2013; Vargas, 2013)

\section{A. Descripción general del sistema DRM}

El sistema DRM está diseñado para ser usado en cualquier frecuencia debajo de los $30 \mathrm{MHz}$, es decir dentro de las bandas de transmisión de onda larga, onda media y onda corta, con las limitaciones de canalización y las variables condiciones de propagación a lo largo de estas bandas. Están disponibles diferentes modos de transmisión que satisfacen estas limitaciones de operación. Cada modo se define por parámetros de transmisión. (ETSI, 2014) (Pérez, 2018)

\section{B. Arquitectura del Estándar DRM}

La Figura 1, representa las etapas de la arquitectura de DRM para el transmisor. A la entrada del diagrama se tiene dos tipos de información:

- Audio y datos codificados que se combinan en el multiplexor de servicio principal, con diferentes relaciones de protección.

- Canales de información denominados Canal de Acceso Rápido (FAC) y Canal de Descripción del Servicio (SDC) que no ingresan al multiplexor.

- El multiplexador combina los niveles de protección de los servicios de datos y de audio.

Figura 1. Arquitectura del Estándar DRM

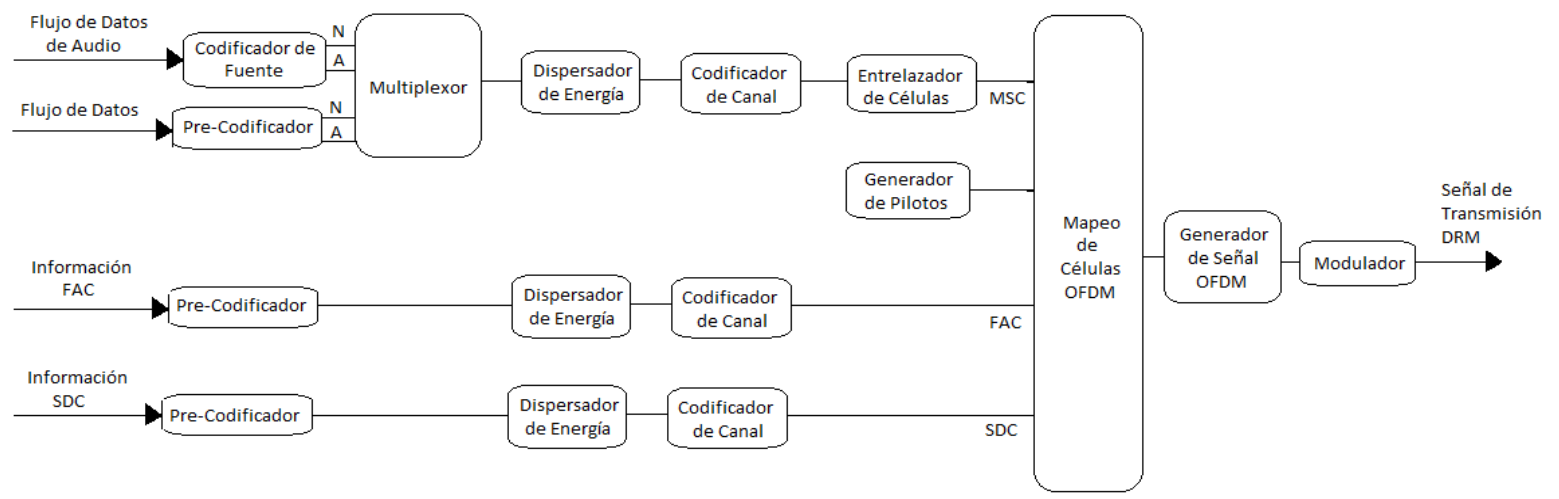

Fuente: (Gil \& Santillán, 2015; Henkel, 2008; Pérez, 2018; Wunsch \& Elsner, 2012).

\section{Metodologia.}

La configuración del transmisor DRM está determinada por una gran cantidad de parámetros, estos dependen uno de otros, siendo en ciertas circunstancias una estructura compleja; siguen una configuración jerárquica, lo cual permite la definición de múltiples escalas desde bajo 
nivel como como la duración del símbolo y la duración del intervalo de guarda hasta otros de orden superior. Es posible definir un grupo pequeño de parámetros definidos por el usuario que permiten una configuración completa del transmisor, los cuales se detallan a continuación: (Gil \& Santillán, 2015; Pérez, 2018; Wunsch \& Elsner, 2012)

\section{- $\mathrm{RM}$}

- $\mathrm{SO}$

- Bandera UEP para una alta protección de bytes

- Tipo de código de audio y velocidad de muestreo de la entrada de audio

- Esquema de mapeo MSC y SDC

- Velocidad de código MSC y SDC

- Bandera de entrelazado: corta o larga

- Nombre de la estación y mensaje de texto

En este proyecto el codificador está implementado para el modo de robustez A, B, C con un valor de Ocupación del Espectro igual a 2,3 y 4 lo que corresponde a un ancho de banda de 5,10 y $20 \mathrm{kHz}$ respectivamente, y utilizando únicamente EEP. Para el mapeo de los canales MSC, SDC, FAC se utiliza el mapeo estándar con 16-QAM para el MSC y 4-QAM para el SDC y FAC. La tasa de código es de 0.5. El entrelazado de células se establece en modo largo. (Pérez, 2018)

La Figura 2, muestra los bloques de configuración de los parámetros de nivel superior iniciales del sistema, desarrollado en GNU Radio para la transmisión de la señal con modulación en DRM. (Pérez, 2018)

Figura 2. Parámetros iniciales configurados
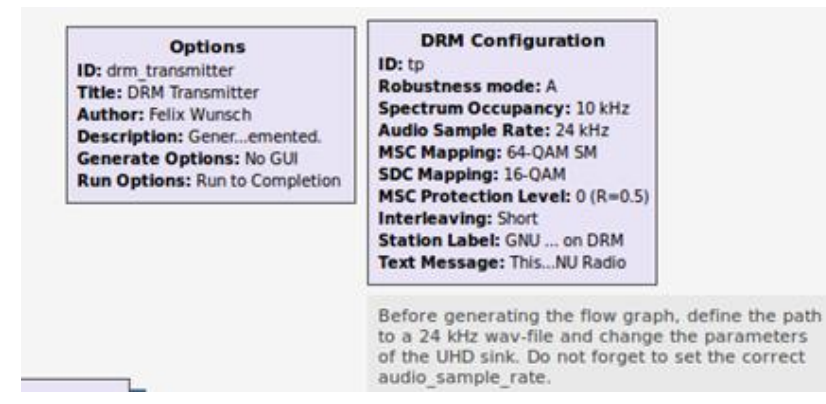

Fuente: (Pérez, 2018)

Una vez definidos los parámetros iniciales del sistema de transmisión, se genera la información que va a ser enviada por cada trama de datos MSC, SDC y FAC. La información del MSC la compone la fuente y la codificación de audio, mientras que las otras dos tramas son generadas automáticamente, ver Figura 3. (Pérez, 2018) 
Figura 3. Tramas MSC, SDC y FAC

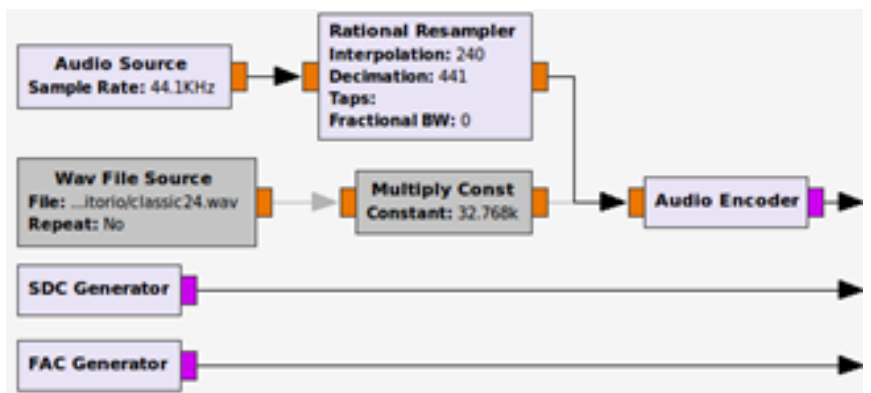

Fuente: (Pérez, 2018)

Fuente de audio: El canal MSC del codificador requiere como entrada un archivo de audio sin comprimir, es decir archivos en formato wave (.WAV). Para los canales FAC y SDC, las entradas requeridas siguen el formato establecido por el estándar, ver Figura 3. (Gil \& Santillán, 2015; Pérez, 2018)

Codificación de audio: DRM utiliza los algoritmos de un subconjunto del estándar MPEG-4 para la codificación de audio. Existe una codificación para la música y dos para la voz. El subconjunto MPEG-4 AAC, que incluye herramientas de robustez contra errores para transmisiones en audio mono y estéreo. El subconjunto de MPEG-4 CELP, que codifica muestras de voz para así tener una mayor robustez en la transmisión de voz en mono, usado en los casos cuando se necesita una baja tasa de bits o se necesita alta robustez a los errores. El subconjunto de MPEG-4 HVXC, que codifica muestras de voz para tasas de bits muy bajas, lo que provee alta robustez a errores de transmisión de voz en mono. El estándar DRM utiliza el formato AAC para comprensión y codificación de audio el cual consta de dos modos: AAC y xHE-AAC. Estos modos se combinan con las herramientas SBR y PS con el objetivo de obtener una comprensión de mayor calidad, ver Figura 3. (Gil \& Santillán, 2015; Pérez, 2018)

Multiplexor: El multiplexor combina las transmisiones de audio y datos a un solo flujo de bits. En esto proceso, los servicios de datos pueden colocarse en la parte protegida superior o inferior, lo que permite protección contra errores para diferentes servicios. En el caso de la aplicación se enviará la información del texto y nombre de la estación transmisora, ver Figura 4. (Gil \& Santillán, 2015; Pérez, 2018)

Figura 4. Datos enviados en la transmisión

$\begin{array}{ll}\text { Station Label } & \text { "CNU Radio on DRM" } \\ \text { Text Message } & \text { "This is a DRM transmission with GNU Radio" }\end{array}$

Fuente: (Pérez, 2018) 
Se añaden nuevos bloques que procesan la información de cada trama por separado. Estos bloques son el Scrambler y el MLC Factory Block, el primero realiza las funciones de dispersador de energía, mientras que el segundo la codificación del canal, ver Figura 5. (Gil \& Santillán, 2015; Pérez, 2018)

Figura 5. Dispersador de energía y Codificación de canal

Fuente: (Pérez, 2018)

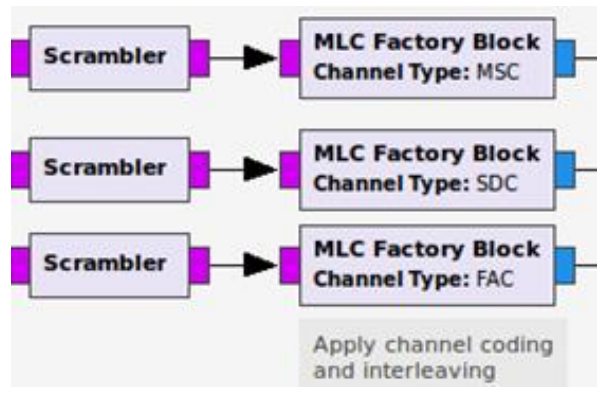

Dispersador de energía: Como los patrones sistemáticos pueden dar como resultado una regularidad no deseada que afecta la señal transmitida o el procesamiento de la señal de forma negativa, los bits se codifican mediante la adición de una secuencia aleatoria de bits o PRBS, ver Figura 6. El objetivo de esta secuencia es romper cadenas largas de constantes o ceros para crear una distribución uniforme entre los bits. (Gil \& Santillán, 2015; Pérez, 2018)

Figura 6. Generación de PRBS con el uso de shift registers

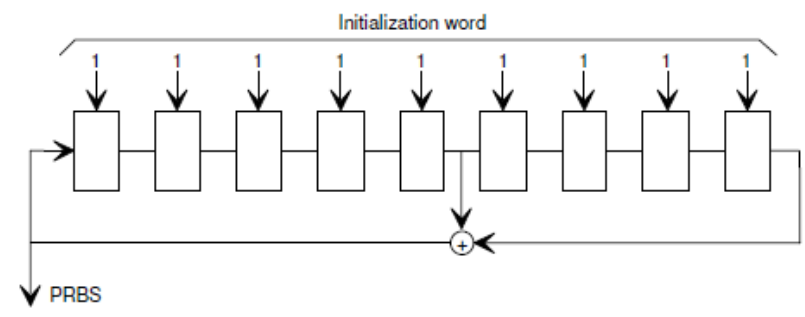

Fuente: (Pérez, 2018)

Codificación de canal: Al utilizar QAM el estándar DRM las posiciones de bits más propensos a tener errores consiguen mayor protección. Una decodificación eficiente es necesaria para que los datos sean recibidos correctamente, siempre que exista una diferencia resultante en las relaciones de señal a ruido en los canales MSC y SDC. Esta diferencia se 
lleva a cabo cuando el MSC utiliza 64-QAM y el SDC 16-QAM, de la misma forma cuando el MSC utiliza 16-QAM y el SDC 4-QAM. (Gil \& Santillán, 2015; Pérez, 2018)

Entrelazado de células: El entrelazado de células solo se usa para el MSC y realiza un entrelazado en el dominio de frecuencia, ver Figura 7. (Gil \& Santillán, 2015; Pérez, 2018)

Figura 7. Entrelazado de células

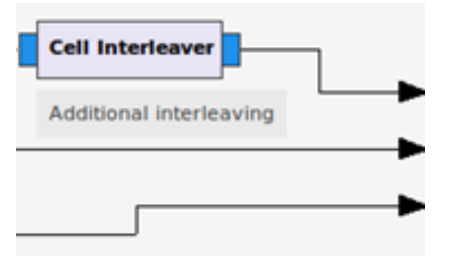

Fuente: (Pérez, 2018)

El principio del entrelazado de células se puede apreciar en la Figura 8. Utiliza el mismo algoritmo de entrelazado que el entrelazado de bits, pero también ofrece un modo "entrelazado largo". En este modo, se utiliza un esquema convolucional para aumentar la robustez de transmisiones en canales que sufren un desvanecimiento severo selectivo en tiempo y frecuencia como es típico para frecuencias inferiores a $30 \mathrm{MHz}$. El retraso del entrelazado siempre es un número entero múltiplo de la longitud de un cuadro de transmisión. DRM30 usa un factor $\mathrm{D}=5 \mathrm{y}$ para $\mathrm{DRM}+$ un valor $\mathrm{D}=6$. Se determina un tiempo como compensación óptima entre el rendimiento y retraso de procesamiento; en el caso de DRM30, esto resulta en un retraso total de 2.4 segundos, ver Figura 9. (Pérez, 2018)

Figura 8. Entrelazado en tiempo y frecuencia

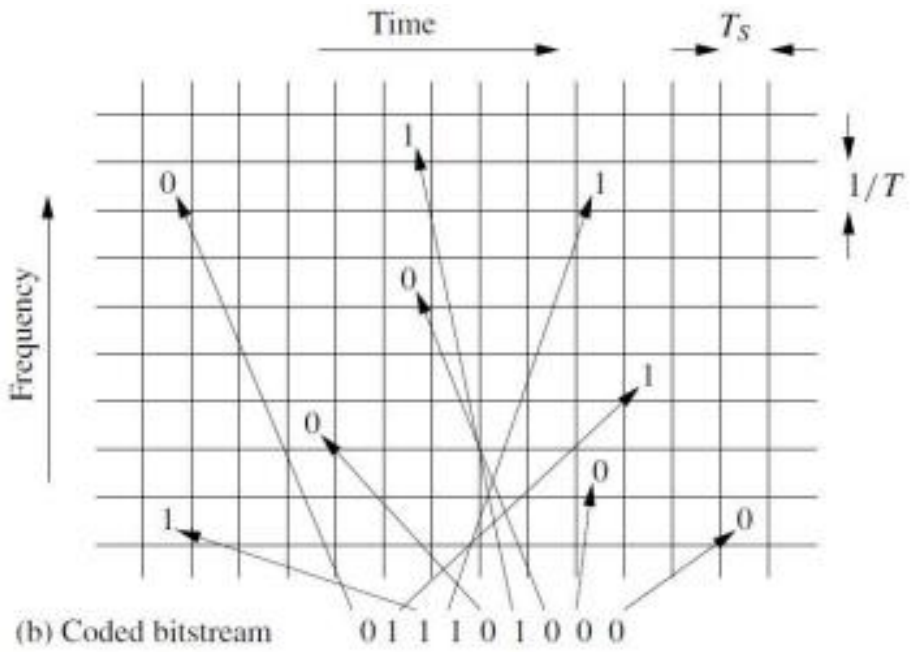

Fuente: (Pérez, 2018) 
Figura 8. Entrelazado en tiempo y frecuencia $X$

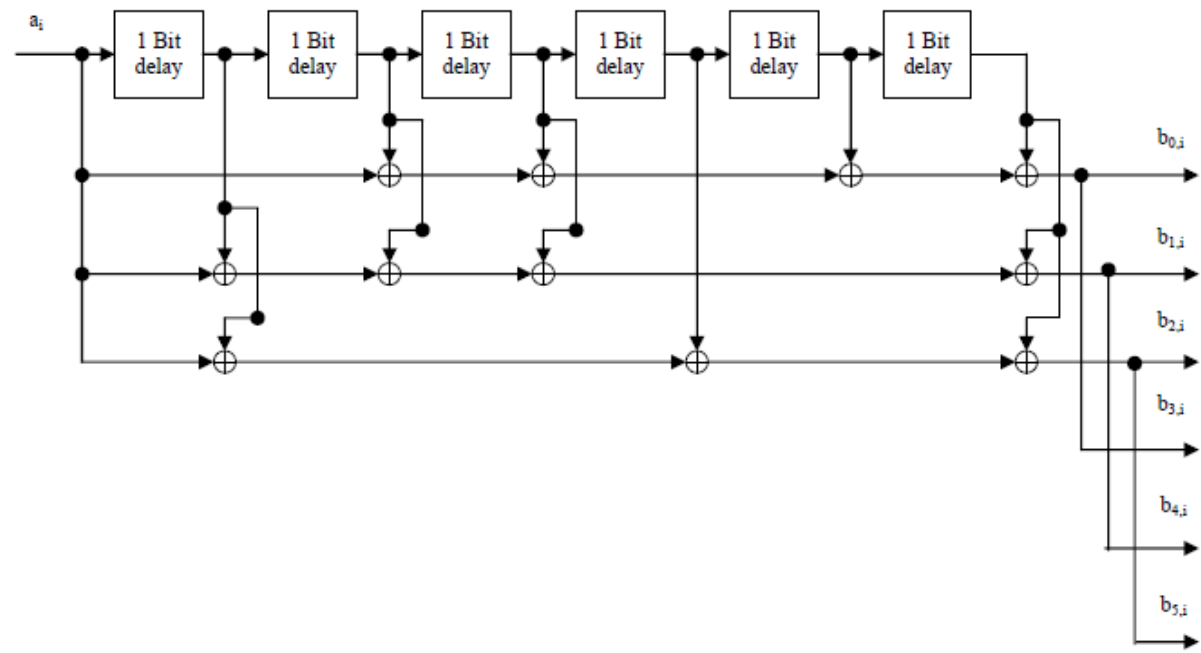

Fuente: (Pérez, 2018)

Mapeo de células OFDM: El mapeo de células OFDM coloca las células MSC, SDC y FAC en el plano de tiempo-frecuencia llamada trama de transmisión. Una trama de transmisión consiste en una concatenación de un cierto número de símbolos OFDM que está determinado por la elección del RM. Además, múltiples tramas de transmisión se agrupan en una supertrama de transmisión, ver Figura 10. (Gil \& Santillán, 2015)

Figura 10. Mapeo de Células OFDM y Células Piloto

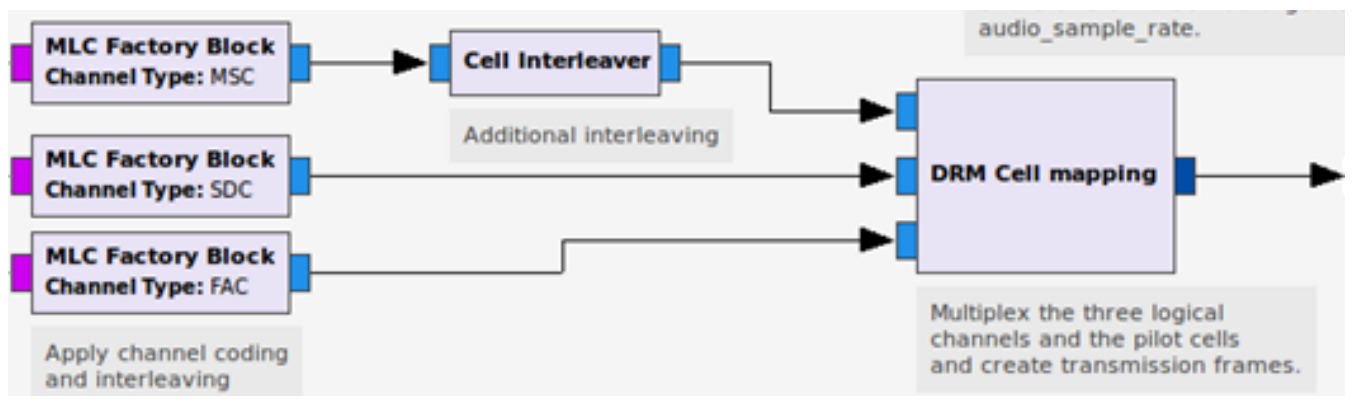

Fuente: (Pérez, 2018)

Además de los tres canales, hay cuatro diferentes símbolos piloto o de referencia que ayudan en la demodulación del receptor. Las células de referencia de frecuencia representan ondas sinusoidales continuas a $750 \mathrm{~Hz}, 2250 \mathrm{~Hz}$ y $3000 \mathrm{~Hz}$. Se utilizan para la detección de una señal DRM y corrección de desplazamiento de frecuencia. Estas las células no están presentes en RM E, DRM +. (Pérez, 2018; Sánchez \& Sagbay, 2013)

Generación de señal OFDM: Para convertir la señal del dominio de frecuencia al dominio de tiempo, se utiliza la Transformada Discreta de Fourier Inversa (IDFT) o su versión 
computacional más eficiente, Transformada Rápida de Fourier Inversa (IFFT), ver Figura 11. (Jara, 2005; Pérez, 2018)

Figura 11. Generación de señal OFDM audio sample rate.

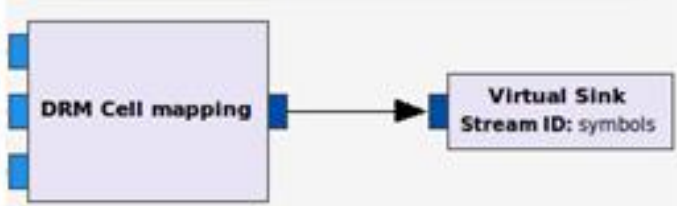

Multiplex the three logical channels and the plot cells and create transmission frames.

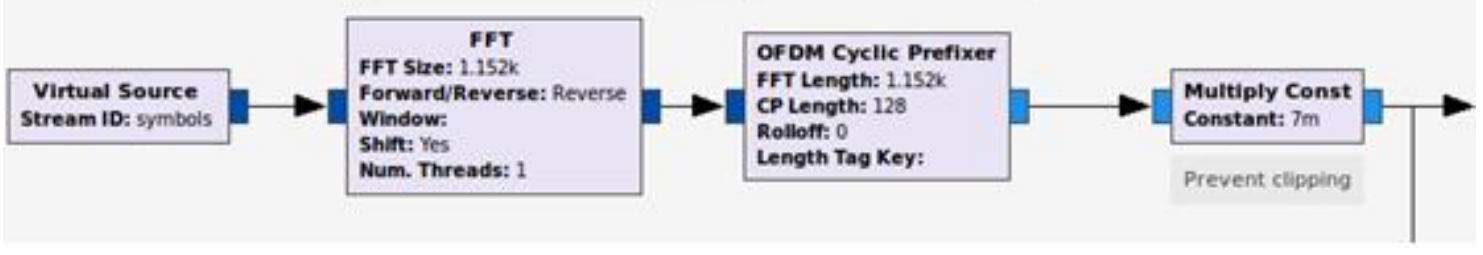

Fuente: (Pérez, 2018)

Para OFDM también se inserta un prefijo cíclico para el manejo de varios trayectos. La duración varía con la elección del RM. En DRM30, puede ser de hasta 7.33 ms de duración y por lo tanto cubrir trayectos múltiples con una longitud de $2200 \mathrm{~km}$. El prefijo cíclico en DRM + está fijado a una duración más corta de $0.25 \mathrm{~ms}$ y, por lo tanto, maneja componentes multitrayecto con una longitud de hasta $75 \mathrm{~km}$. (Pérez, 2018)

\section{Resultados.}

Se desarrolló una interfaz gráfica para la configuración y transmisión de la señal en DRM, siendo mucho más intuitiva para usuarios en general, como se puede ver en la Figura. (Pérez, 2018)

Figura 12. Interfaz GUI DRM Transmitter
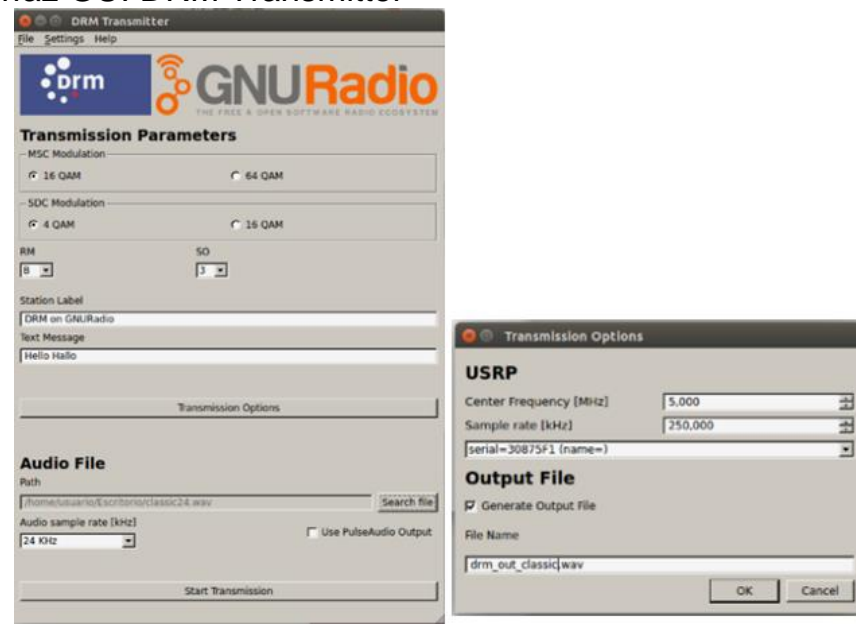

Fuente: (Pérez, 2018) 
En la Figura 13 y en la Figura 14 se puede observar la señal emitida por el transmisor. (Pérez, 2018)

Figura 13. Análisis espectral señal emitida por el transmisor

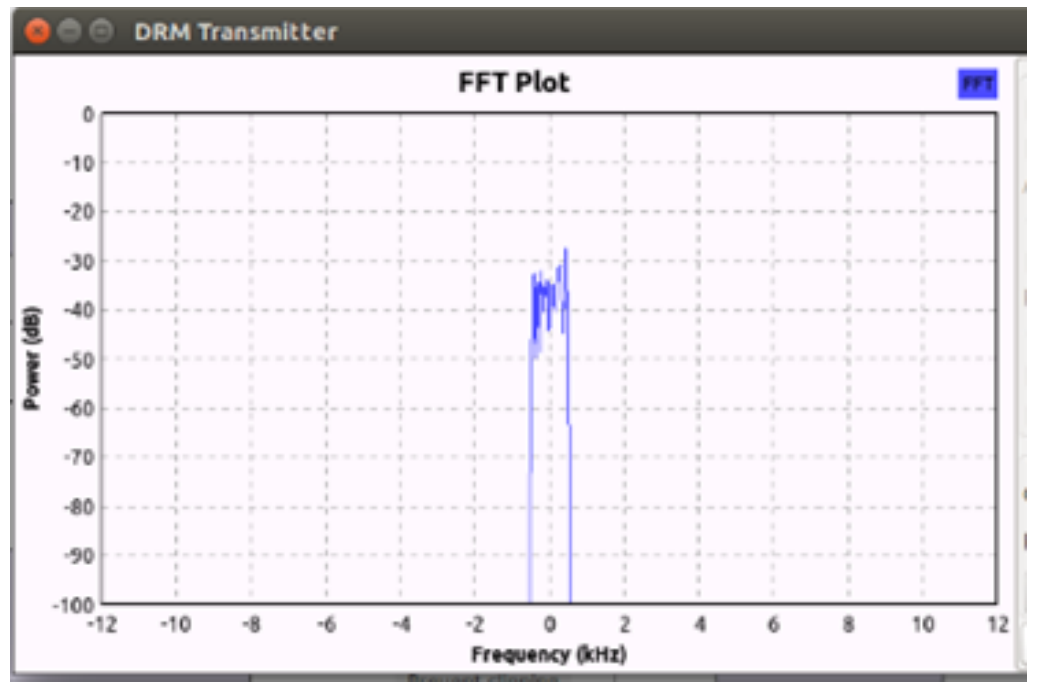

Fuente: (Pérez, 2018)

Figura 14. Análisis espectral señal emitida por el transmisor

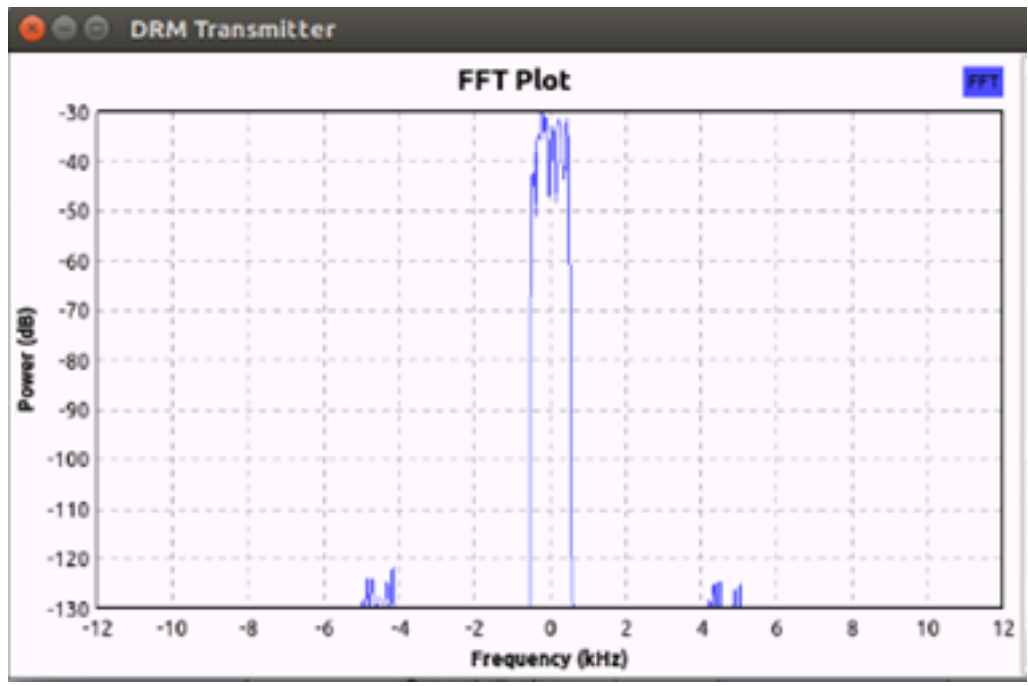

Fuente: (Pérez, 2018)

Para la puesta en marcha del sistema de transmisión se ejecuta el flow graph. Como se puede observar en la Figura 15, en las cuales se realiza la transmisión de una señal generada a través de GNU Radio. (Pérez, 2018) 


\section{(EDigital}

ISSN: 2600-5859

Figura 15. Puesta en marcha del sistema (Execute flow graph)

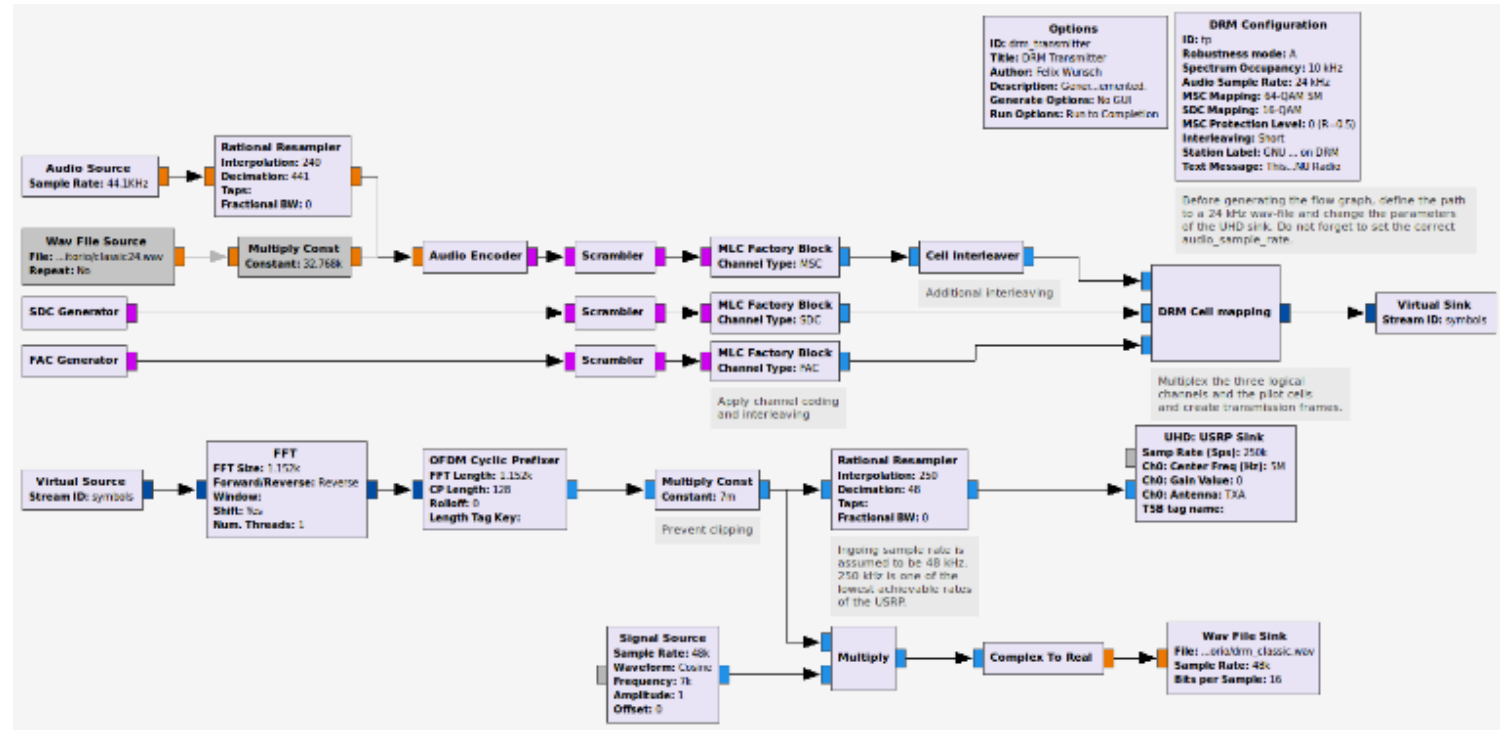

Fuente: (Pérez, 2018)

Para comprobar que la señal se ha transmitido se corrobora con la utilización de un analizador de espectros. Como se puede comprobar en la Figura 16, Figura 17, Figura 18. (Pérez, 2018)

Figura 16. Tarjeta USRP y señal mostrada en el analizador de espectros

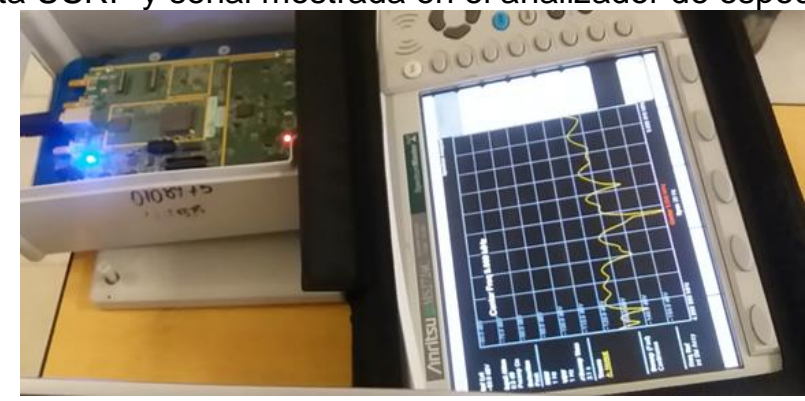

Fuente: (Pérez, 2018)

Figura 17. Señal transmitida, mostrada en el analizador de espectros

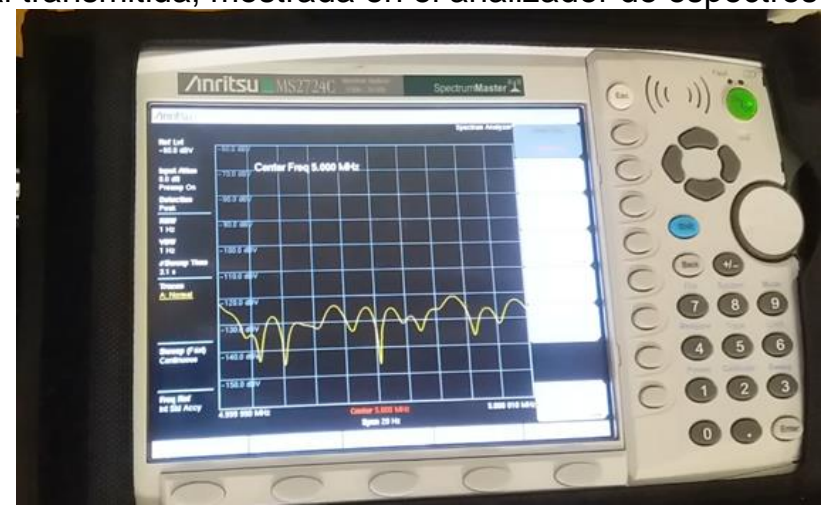

Fuente: (Pérez, 2018) 
Figura 18. Señal transmitida a $5 \mathrm{MHz}$

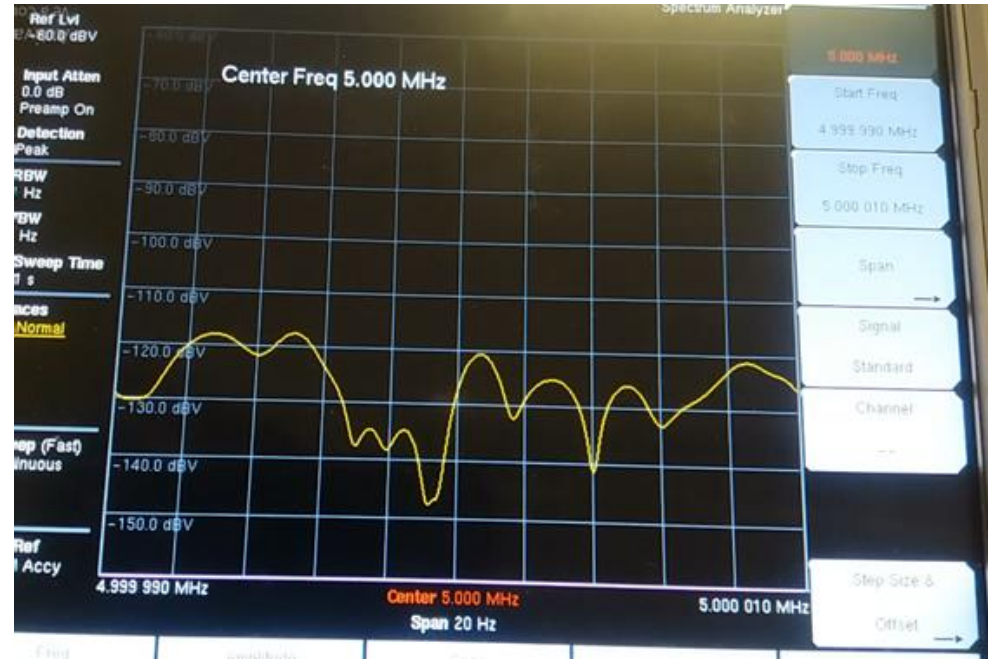

Fuente: (Pérez, 2018)

Complementario a la transmisión de la señal se puede generar un archivo en .WAV codificado y modulado con DRM, para ser procesado a través del software Dream SDR. (Pérez, 2018)

La Figura 19, muestra la pantalla principal del software DREAM en la cual se puede apreciar el nombre de la estación y la codificación del audio. (Pérez, 2018)

Figura 19. Pantalla principal DREAM. Modo A, Ancho de Banda $5 \mathrm{kHz}$

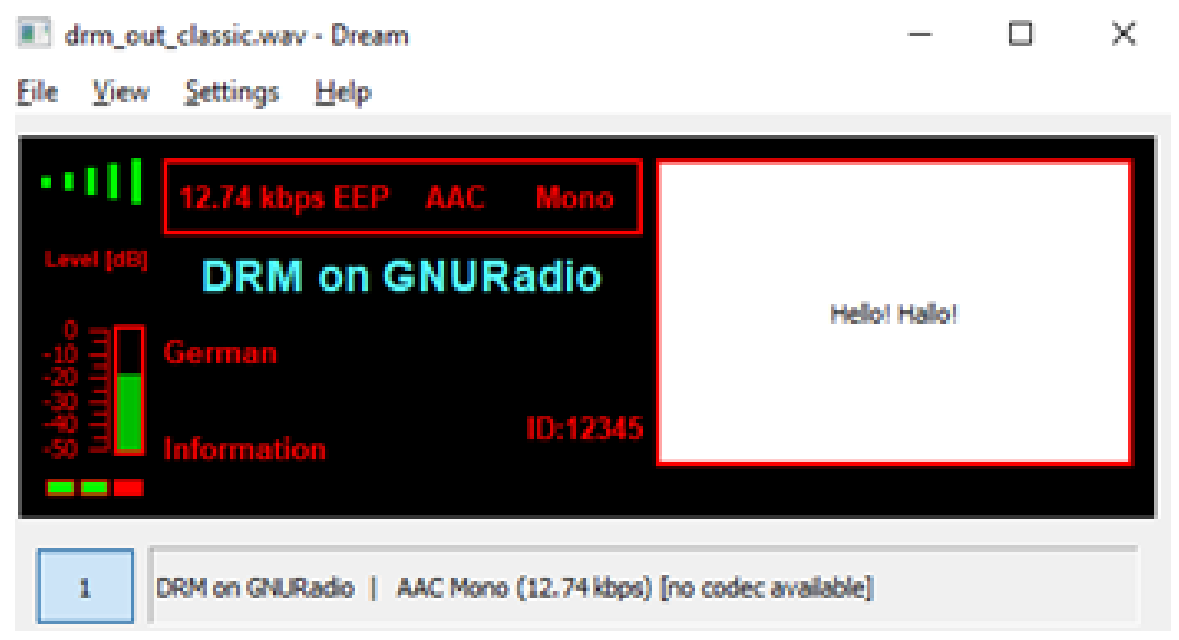

Fuente: (Pérez, 2018)

En la Figura 20, se muestra el análisis espectral de la señal con los niveles de potencia definidos por la longitud de onda o frecuencia. La señal ocupa un ancho de banda de $10 \mathrm{kHz}$. (Pérez, 2018) 
Figura 20. Input Spectrum
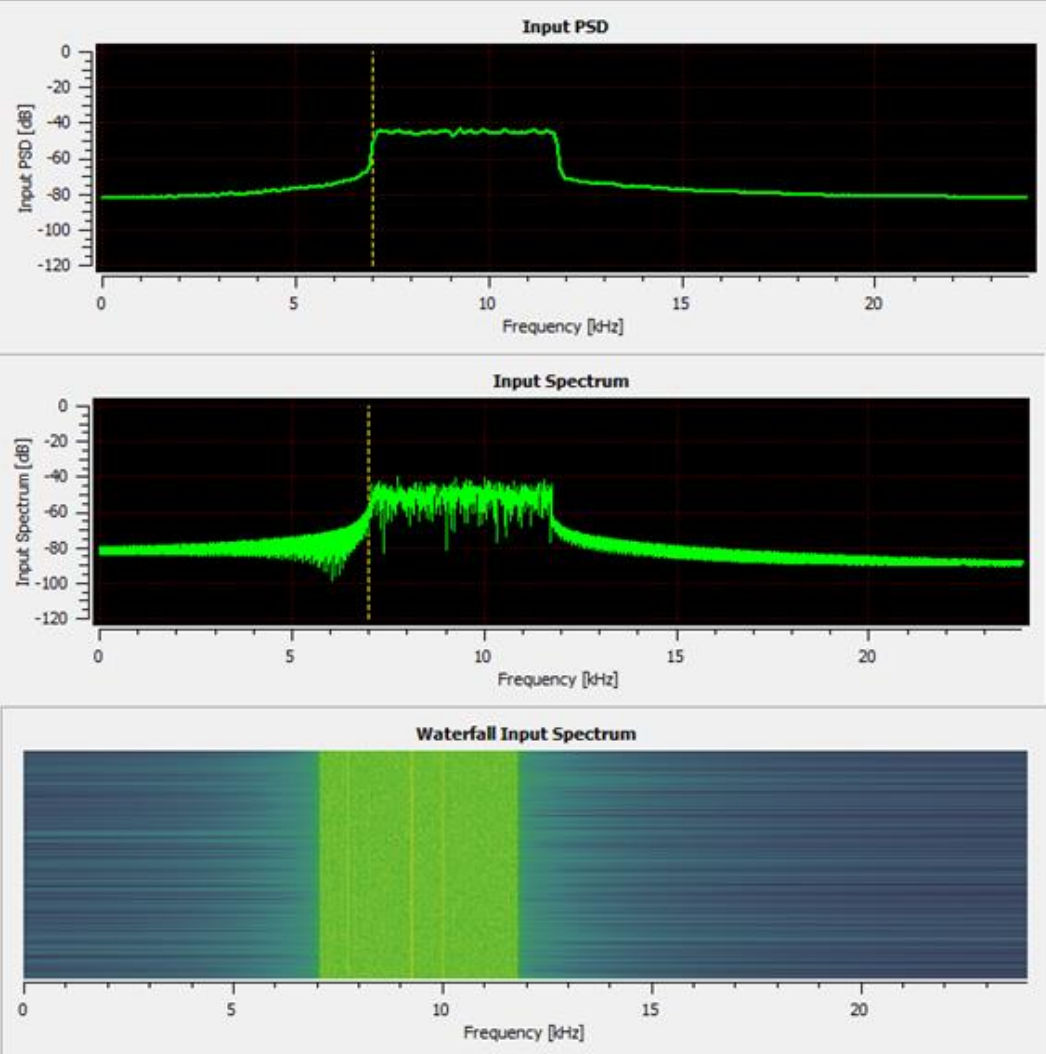

Fuente: (Pérez, 2018)

La señal se mantiene estable en $35.2 \mathrm{~dB}$, siendo posible verificar en la Figura 21. (Pérez, 2018)

Figura 21. Pantalla principal SNR Spectrum. Modo A, Ancho de Banda $5 \mathrm{kHz}$

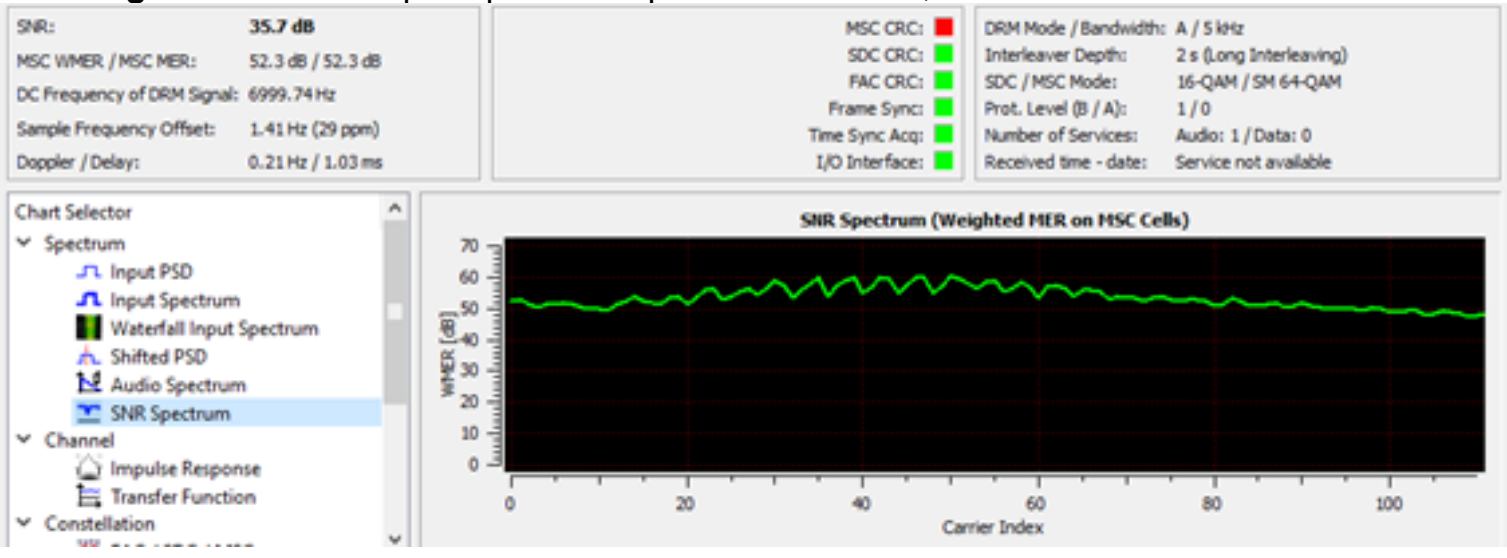

Fuente: (Pérez, 2018)

La Figura 22 muestra el diagrama de constelaciones de los canales MSC, SDC y FAC. (Pérez, 2018) 
Figura 22. Diagrama de constelación de la modulación QAM

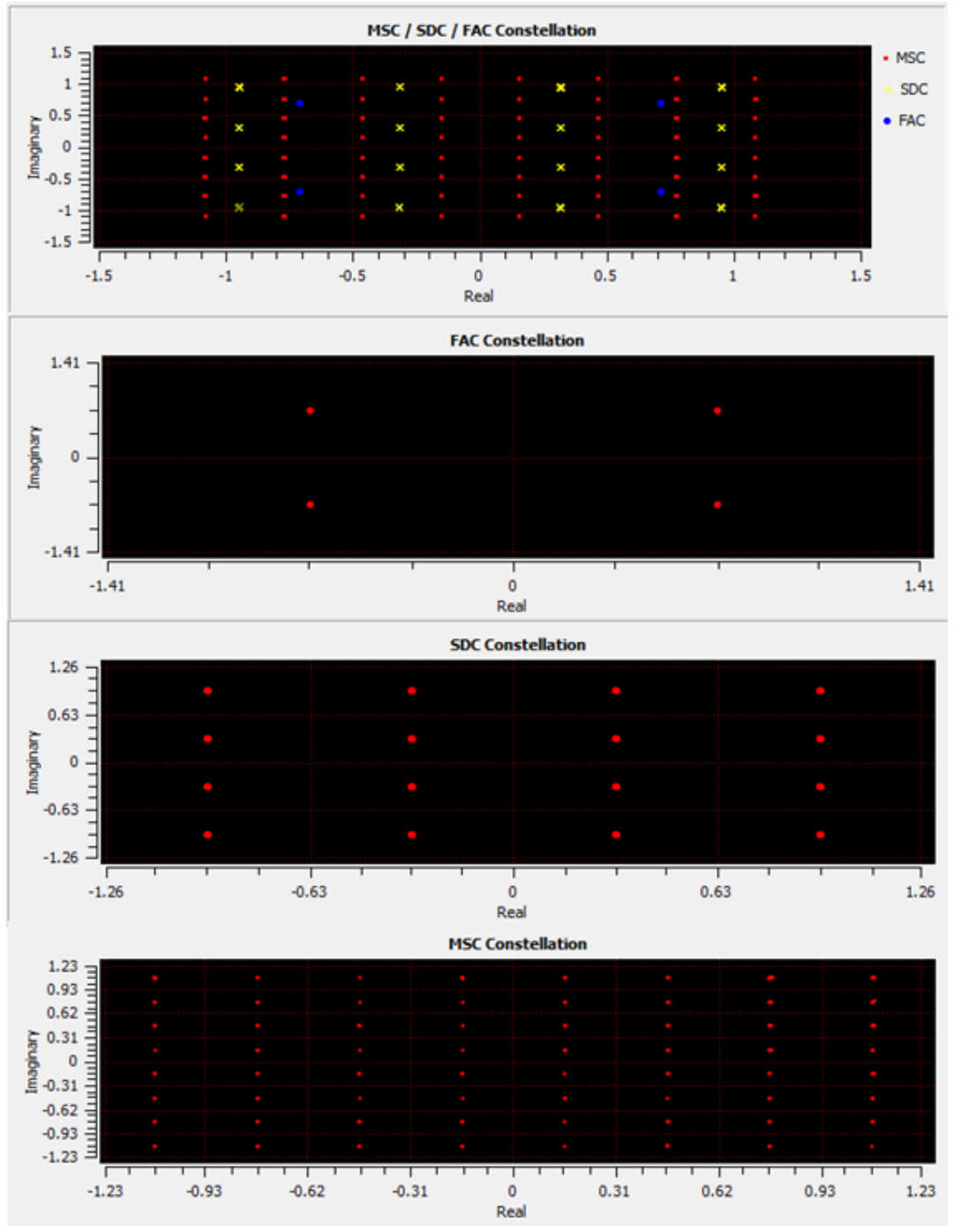

Fuente: (Pérez, 2018)

Los tres modos de robustez configurados tuvieron una variación pequeña referente a cada parámetro de velocidad de transmisión y relación de señal a ruido. Con una ocupación espectral de 3, una tasa de código de 0.5, un entrelazado largo, y una modulación en MSC de 64-QAM y en SDC de 16-QAM, un ancho de banda de 10kHz se obtuvo (23.62 kbps y 17.3 dB en Modo A), (17.12 kbps y 21.50 dB en Modo B), (10.08 kbps y 14.05 dB en Modo C), podemos observar en base a los resultados que las características de funcionamiento de cada modo de robustez para un determinado canal de transmisión varían los valores de la velocidad de transmisión y la relación señal a ruido, ofreciendo el modo B una mejor protección de la señal con respecto al ruido, mientras que el modo A ofrece mayor velocidad de transmisión, ver Tabla 1. (Pérez, 2018) 
ISSN: 2600-5859

Vol. 3, N².2, p. 169-187, junio, 2020

www.concienciadigital.org

Tabla 1. Resumen Datos obtenidos por el sistema.

\begin{tabular}{cccccc}
\hline Modo & $\begin{array}{c}\text { Ancho de } \\
\text { banda (kHz) }\end{array}$ & $\begin{array}{c}\text { MSC } \\
\text { X-QAM }\end{array}$ & $\begin{array}{c}\text { SDC } \\
\text { X-QAM }\end{array}$ & $\begin{array}{c}\text { Velocidad } \\
\text { (kbps) }\end{array}$ & SNR (dB) \\
\hline A & 5 & 64 & 16 & 12.74 & 35.7 \\
A & 5 & 16 & 4 & 8,84 & 68 \\
A & 10 & 64 & 16 & 23.62 & 17.3 \\
A & 10 & 16 & 16 & 18,44 & 71,8 \\
A & 10 & 16 & 4 & 18,44 & 46,3 \\
A & 20 & 64 & 16 & 71.96 & 22.7 \\
B & 5 & 16 & 4 & 6.9 & 26.3 \\
B & 5 & 64 & 16 & 9,92 & 40,8 \\
B & 10 & 64 & 16 & 17.12 & 21.5 \\
B & 10 & 16 & 16 & 14.56 & 17.7 \\
B & 10 & 16 & 4 & 14.56 & 23.6 \\
B & 20 & 64 & 16 & 42,9 & 34,3 \\
C & 10 & 16 & 4 & 11,48 & 35 \\
C & 10 & 16 & 16 & 9,18 & 22,4 \\
C & 20 & 64 & 16 & 10,08 & 14,05 \\
\hline
\end{tabular}

Fuente: (Pérez, 2018)

Como resume del análisis del comportamiento de la señal frente a las distintas características de los medios de robustez y los parámetros iniciales del transmisor se adjunta la Grafica 1 y la Grafica 2en que se observa el comportamiento de la velocidad de transmisión y la relación de señal a ruido (SNR) de los tres modos de robustez. (Pérez, 2018)

Grafica 1. Velocidad de transmisión de los tres modos de robustez

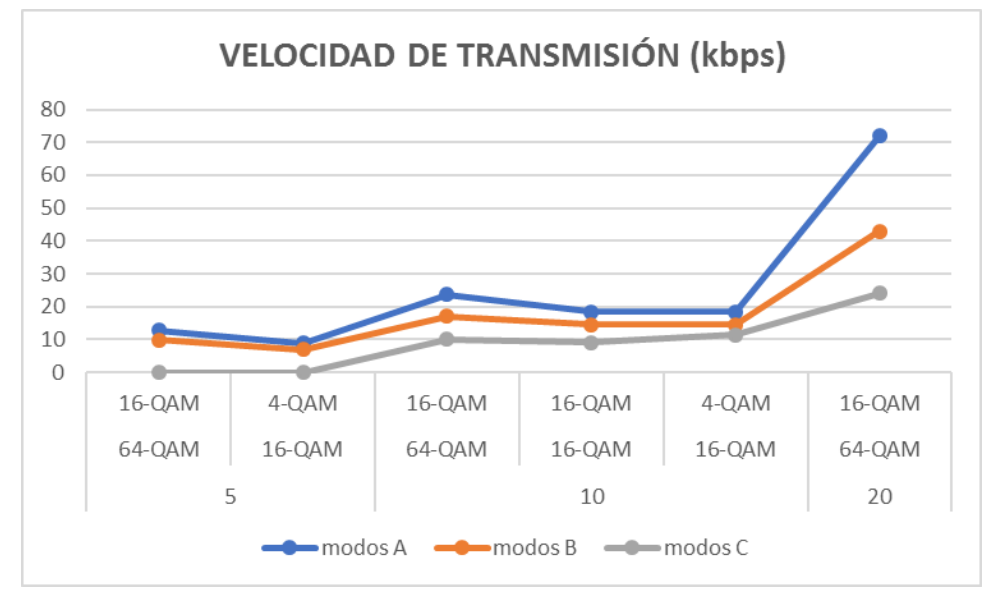

Fuente: (Pérez, 2018) 
Grafica 2. Relación de señal a ruido de los tres modos de robustez

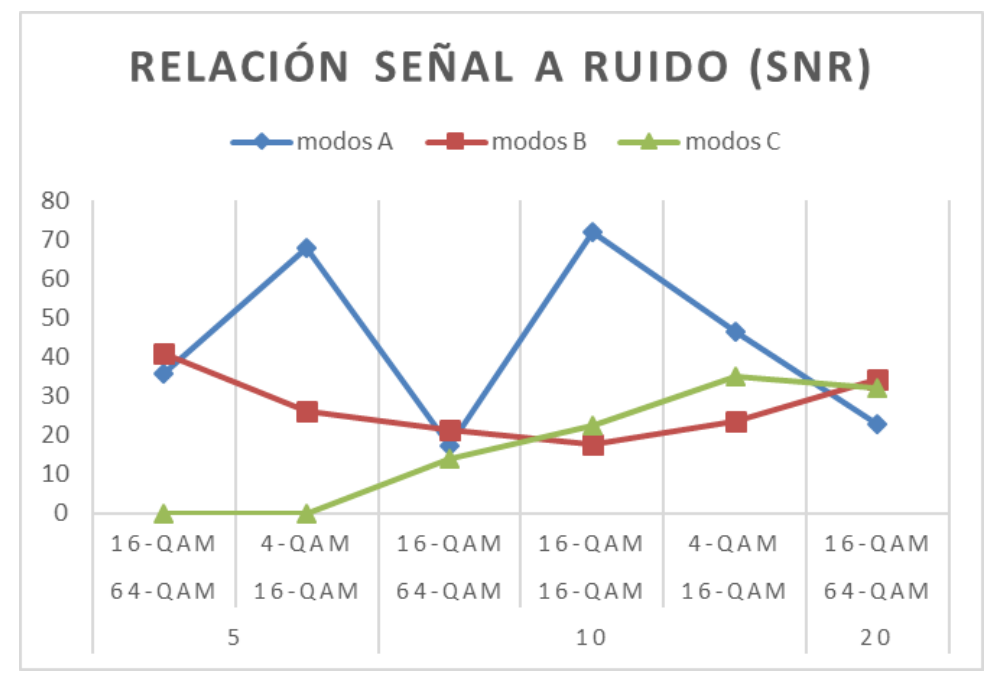

Fuente: (Pérez, 2018)

El comportamiento de la velocidad de transmisión y la relación de señal a ruido son muy similares en los tres modos de robustez, las cuales varían dependiendo el ancho de banda y la modulación QAM escogida. Sin embargo, en una transmisión con un ancho de banda de $20 \mathrm{kHz}$ la velocidad de transmisión se dispara en picos muy altos como se aprecia gráficamente tiene un porcentaje más alto de diferencia de los otros dos. (Pérez, 2018)

El valor de la relación de señal a ruido obtiene un valor alto en un ancho de banda de $5 \mathrm{kHz}$ con una modulación de 16-QAM (MSC) y 4-QAM (SDC), y con un ancho de banda de 10kHz con una modulación de 16-QAM (MSC) y 16-QAM (SDC). Se podría concluir que es el mejor modo de robustez para la transmisión de señales es el modo de robustez A. La modulación QAM con mayor estabilidad en el sistema es en el canal MSC de 16-QAM y en el canal SDC de 4-QAM, dando mejores resultados para la trasmisión de la señal. (Pérez, 2018)

\section{Conclusiones.}

- Se ha diseñado e implementado un sistema de transmisión de AM digital a través del estándar DRM para determinar la calidad de servicios de la emisión de las señales en diferentes condiciones del medio, con lo cual se determinó la fidelidad en la recepción de señales digitales.

- Se ha determinado que los valores de la velocidad de transmisión y la relación de señal a ruido varían según el modo de robustez, el ancho de banda y la modulación QAM utilizada. 
- Usando el códec AAC, se requiere un ancho de bando de $5 \mathrm{kHz}$ para realizar una transmisión estable de la señal comparada con la emitida por una estación FM analógica.

- Los modos de robustez se determinaron para ciertas características del medio, siendo el modo A, el más fiable, con una alta velocidad de transmisión y relación de señal a ruido, lo cual determina que se puede utilizar el estándar DRM para transmisiones en media y baja frecuencia.

- El sistema DRM emplea las mejores tecnologías de compresión de sonido ya que usa los algoritmos de audio y voz del MPEG-4 que brinda excelente calidad de sonido a bajas tasas de bit como la codificación AAC, las codificaciones de voz CELP y HVXC, complementada con Replicación de Banda Espectral SBR como su codificación digital principal. La SBR mejora la calidad de audio percibida y también puede emplear la codificación Estéreo Paramétrica para las señales estéreo.

- El trabajo más eficiente del sistema se realizó con los parámetros configurados con el modo A de robustez, la ocupación del espectro en 3, un ancho de banda de $10 \mathrm{kHz}$, una tasa de código de 0.5 , un entrelazado largo y una modulación para el canal MSC de 16-QAM y para el canal SDC de 4-QAM.

- La calidad total de recepción y fidelidad de servicio de recepción portátil y móvil serán similares a la actuación en receptores fijos. El sistema cubre los efectos del multi-camino y las características de cambios de propagación presentes en transmisiones de onda larga, media y onda corta

\section{Referencias bibliográficas.}

Erazo, H. J. (2009). Estudio y Análisis de la Tecnología de Recursos de Frecuencia única, y su Aplicacion en la Radiodifucion en las Bandas de AM Y FM para la Optrimización del Espectro Electromagnético en la Ciudad de QUito. In Escuela Politécnica Nacional. http://bibdigital.epn.edu.ec/bitstream/15000/1451/1/CD-2125.pdf

ETSI. (2014). ES 201980 - V3.1.1 - Digital Radio Mondiale (DRM); System Specification. Intellectual Property, 195.

https://www.etsi.org/deliver/etsi_es/201900_201999/201980/04.01.01_60/es_201980v 040101p.pdf

Gil, P. D., \& Santillán, F. G. (2015). Implementación de las Etapas de Codificación, Mapeo y Mapeo Inverso QAM de un Transmisor de Radio Digital Según el Estándar DRM30. In Universidad de Cuenca. https://doi.org/10.1017/CBO9781107415324.004

Henkel, M. (2008). Architektur eines DRM-Empfängers und Basisbandalgorithmen für Frequenzakquisition und Kanalschätzung. Institut Für Nachrichtentechnik Universität Karlsruhe (TH), 171. http://d-nb.info/998336947/34

Jara, A. E. (2005). Multiplexación por División de Frecuencia Ortogonal Codificada ( Cofdm ). In Universidad Austral de Chile. http://cybertesis.uach.cl/tesis/uach/2005/bmfcij.37m/doc/bmfcij.37m.pdf

Pérez, J. J. (2018). Estudio y Análisis de la Calidad de Servicio en la Transmisión Am Digital Mediante el Uso de Códecs en Usrp [Escuela Superior Politécnica de Chimborazo]. In Escuela Superior Politécnica De Chimborazo. http://dspace.espoch.edu.ec/bitstream/123456789/9283/1/20T01111.PDF

Puente, M. F. (2005). Implementacion del Servicio de Radiodifusion Digital Drm ( Digital 
Radio Mondiale ) en el Ecuador [ESPE]. In ESPE. https://repositorio.espe.edu.ec/bitstream/21000/2477/1/T-ESPE-027548.pdf

Salinas, M. A. (2011). Comparación de tecnologías de radio digital para su aplicación en México. In Universidad Nacional Autónoma de México. http://www.ptolomeo.unam.mx:8080/xmlui/bitstream/handle/132.248.52.100/161/A1. pdf? sequence $=1$

Sánchez, F., \& Sagbay, G. (2013). Estudio y Análisis de la Estandarización y Regulación para la migración del sistema de radio analógico al digital en el Ecuador. Universidad Politécnica Salesiana, 228. dspace.ups.edu.ec/bitstream/123456789/5253/1/UPSCT002755.pdf

Vargas, L. P. (2013). Diseño de Una Radiodifusora Digital en Base al Estándar Drm (Digital Radio Mondiale) Para la Banda de Am. In Escuela Politecnica Nacional. https://books.google.com.ec/books?id=yIAzAQAAMAAJ\&pg=PA130\&dq=enzima+p apina\&hl=es-419\&sa=X\&ved=0ahUKEwjQMbzjvfPAhVC7iYKHYM7A30Q6AEIQTAH\#v=onepage\&q=enzima papina\&f=false Wunsch, F., \& Elsner, M. J. (2012). Implementation of a DRM + transmitter in the GNU Radio software radio framework Introduction. http://static1.1.sqspcdn.com/static/f/679473/20463131/1349016358370/wunschgrcon12_gsoc_drm.pdf?token=IuVNQsWDcvePIw22CZ9A8VHdl1s\%3D 


\section{PARA CITAR EL ARTÍCULO INDEXADO.}

Pérez Insuasti, J. J., Zabala Haro, M. A., Pazmiño Armijos, A. O., \& Moreno Avilés, H. O. (2020). Análisis de calidad de servicio en la transmisión por AM digital mediante el uso de códecs en USRP . ConcienciaDigital, 3(2.2), 169-187. https://doi.org/10.33262/concienciadigital.v3i2.2.1254

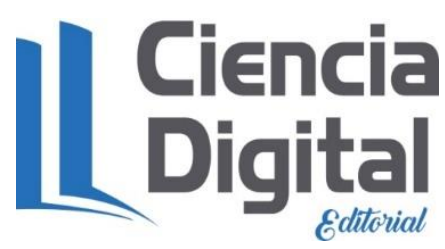

El artículo que se publica es de exclusiva responsabilidad de los autores y no necesariamente reflejan el pensamiento de la Revista Conciencia Digital.

El artículo queda en propiedad de la revista y, por tanto, su publicación parcial y/o total en otro medio tiene que ser autorizado por el director de la Revista Conciencia Digital.

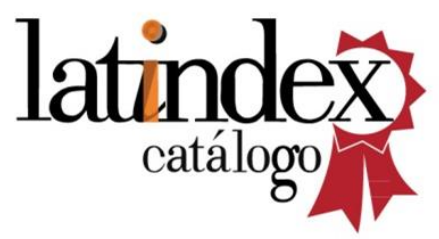

